\title{
Palliation of jaundice in pancreatic cancer: stent or surgery?
}

\author{
H. Ramesh
}

Received: 7 February 2013 / Accepted: 7 February 2013 /Published online: 17 March 2013

(C) Indian Society of Gastroenterology 2013

Pancreatic cancer still remains a lethal disease, and a large proportion of patients present for treatment at an advanced stage. Surgical resection is still the best hope for cure, but is only possible in localized disease. Locally advanced tumors may benefit from en bloc resection of the superior mesenteric vein or adjacent portal vein, and neoadjuvant chemoradiation may help to render some advanced tumors resectable. For the majority of other locally advanced or disseminated pancreatic head cancers, palliation is the only realistic option. Patients may suffer from obstructive jaundice, with or without cholangitis, and disabling pruritus, from vomiting due to obstruction to the gastric outlet, and other symptoms such as anorexia or abdominal pain.

What is the optimal approach to palliating patients with obstructive jaundice due to pancreatic cancer? Patients may be classified into two groups: those with obviously unresectable or metastatic disease on preoperative evaluation or with severe comorbid illness which precludes operation or renders it at high risk; and those where preoperative imaging indicates a possibly resectable pancreatic tumor.

In patients where evaluation has established that the tumors or patients are unsuitable for surgical resection, the decision to employ nonsurgical palliation is more straightforward. Expandable metallic stent insertion provides effective palliation of jaundice, duodenal stents may be used for gastric outlet obstruction (although these may be difficult to place when the obstruction extends to the third part of the duodenum), and endoscopic ultrasound-guided or percutaneous celiac plexus neurolysis may be employed for relief of intractable pain. In this issue of the Journal, Nikfarjam et al. [1] have compared the outcomes following stent insertion vs. surgical bypass. The "majority" of their patients who

\section{H. Ramesh $(\bowtie)$}

Department of Surgical Gastroenterology and Liver

Transplantation, Lakeshore Hospital and Research Center, Cochin 682 024, India

e-mail: hramesh@vsnl.com underwent surgical bypass were candidates for curative resection which were found to be unresectable on the operation table. Stents were used in older patients and those with poor performance status and deeper jaundice. Overall, survival figures were comparable, though stent insertion was followed by a significantly larger number of reinterventions, and $13 \%$ required subsequent surgical intervention. Forty-one percent of metallic stents blocked over a median follow up of 224 days. The paper highlights a number of interesting observations: (a) patients who underwent stenting were less likely to undergo palliative chemotherapy-presumably their general condition was too poor to allow the use of chemotherapy; (b) the majority of stent blockage could be treated by restenting, and the chief area of failure was the development of duodenal obstruction; and (c) the only factor affecting survival was the presence of metastatic disease. Patients without metastatic disease may have significant 12-month survival during which re-interventions may be necessary. Does this mean that patients who have locally advanced, obviously unresectable disease but without metastases must be subjected to operative bypass rather than endoscopic stenting? The data in their paper are not strong enough to suggest such an approach, and other publications have shown that $97 \%$ of patients who are treated by endoscopic palliation never require a surgical procedure until their death [2]. This treatment policy has also been supported by decision analysis [3] and a meta-analysis [4]. It is safe to conclude therefore that patients with obviously unresectable tumors or those unfit for surgery are preferably treated by endoscopic stent placement, though a variable number of patients in this group may live long enough to necessitate reinterventions.

The second clinical situation, where a tumor is deemed unresectable at laparotomy, presents a much more vexing issue. Should these patients undergo closure of laparotomy wounds and be scheduled for a later endoscopic stent 
placement, or should an operative bypass be added? Although improved imaging has allowed a more true preoperative estimation of resectability [5], there still exists a borderline group where surgical exploration is the only method of confirming resectability. Diagnostic laparoscopy is now an accepted modality which helps to identify small hepatic or peritoneal metastases, as these are not identified by preoperative imaging [6]. Every effort must be made to avoid a non-therapeutic laparotomy as it has been accompanied by poor outcomes in terms of morbidity, decreased likelihood of receiving systemic treatment, and poor quality of life [7]. Despite these, a significant number of patients are likely to undergo a non-therapeutic laparotomy with unresectability being identified at operation. Should these patients undergo operative bypass, or should they be selected for postoperative stent placement? While the paper by Nikfarjam et al. does not specifically address this problem, they and others [8] report that surgical bypass did not require any other interventions in the postoperative course. The experience of some larger centers has been substantially different (notably from the Memorial Sloan-Kettering group [9]) who have shown that operative bypass (both biliary and duodenal) is followed by a significant number of re-interventions. It appears that an operative bypass is not a panacea in this situation and further may be best performed in specialized centers which deal with curative resection for pancreatic cancer [10]. Overall, it may be prudent to select patients for operative bypass where metastatic disease is not present and leave those with extensive metastases not detected by preoperative imaging for endoscopic therapy.

The emergence of laparoscopic techniques has allowed the possibility of laparoscopic bypass with a potential for lower morbidity, although superiority has not been established over open surgery. Robotic surgery has also striven to make an impact [11].

There have been developments in the choice of stents toocovered stents tended to migrate, whereas uncovered stents ran the risk of tumor in growth [12].

Randomized trials are not feasible because the patient population is heterogeneous, and treatment choices must depend on disease extent and patient performance. In general, it may be inferred that endoscopic palliation would be suitable for patients with shorter life expectancy and surgical palliation for those with locally advanced tumors without metastases and whose performance status is good with consequent longer life expectancy $[13,14]$.

\section{References}

1. Nikfarjam M, Hadj AK, Muralidharan V, et al. Biliary stenting versus surgical bypass for palliation of periampullary malignancy. Indian J Gastroenterol. 2013;32. doi:10.1007/s12664-012-0274-1.

2. Espat NJ, Brennan MF, Conlon KC. Patients with laparoscopically staged unresectable pancreatic adenocarcinoma do not require subsequent surgical biliary or gastric bypass. J Am Coll Surg. 1999;188:649-55.

3. Siddiqui A, Spechler SJ, Huerta S. Surgical bypass versus endoscopic stenting for malignant gastroduodenal obstruction: a decision analysis. Dig Dis Sci. 2007;52:276-81.

4. Moss AC, Morris E, Leyden J, MacMathuna P. Malignant distal biliary obstruction: a systematic review and meta-analysis of endoscopic and surgical bypass results. Cancer Treat Rev. 2007;33: 213-21.

5. Prokesch RW, Schima W, Chow LC, Jeffrey RB. Multidetector CT of pancreatic adenocarcinoma: diagnostic advances and therapeutic relevance. Eur Radiol. 2003;13:2147-54.

6. White R, Winston C, Gonen M, et al. Current utility of staging laparoscopy for pancreatic and peripancreatic neoplasms. J Am Coll Surg. 2008;206:445-50.

7. de Rooij PD, Rogatko A, Brennan MF. Evaluation of palliative surgical procedures in unresectable pancreatic cancer. Br J Surg. 1991;78:1053-8.

8. Lesurtel M, Dehni N, Tiret E, Parc R, Paye F. Palliative surgery for unresectable pancreatic and periampullary cancer: a reappraisal. J Gastrointest Surg. 2006;10:286-91.

9. Lyons JM, Karkar A, Correa-Gallego CC, et al. Operative procedures for unresectable pancreatic cancer: does operative bypass decrease requirements for postoperative procedures and in-hospital days? HPB (Oxford). 2012;14:469-75.

10. Ausania F, Vallance AE, Manas DM, et al. Double bypass for inoperable pancreatic malignancy at laparotomy: postoperative complications and long-term outcome. Ann R Coll Surg Engl. 2012;94:563-8

11. Buchs NC, Addeo P, Bianco FM, Elli EF, Ayloo S, Giulianotti PC. Robotic palliation for unresectable pancreatic cancer and distal cholangiocarcinoma. Int J Med Robot. 2011;7:60-5.

12. Kullman E, Frozanpor F, Soderlund C, et al. Covered versus uncovered self-expandable nitinol stents in the palliative treatment of malignant distal biliary obstruction: results from a randomized, multicenter study. Gastrointest Endosc. 2010;72:915-23.

13. Maosheng D, Ohtsuka T, Ohuchida J, et al. Surgical bypass versus metallic stent for unresectable pancreatic cancer. J Hepato-BiliaryPancreat Surg. 2001;8:367-73.

14. Castano R, Lopes TL, Alvarez O, Calvo V, Luz LP, Artifon EL. Nitinol biliary stent versus surgery for palliation of distal malignant biliary obstruction. Surg Endosc. 2010;24:2092-8. 